\title{
Two types of aa3-nominals in Cantonese
}

\author{
Joanna Ut-Seong Sio and Sze-Wing Tang \\ Palacky University Olomouc The Chinese University of Hong Kong
}

This paper provides an overview of the grammatical properties of the Cantonese $a a_{3}$, a nominal element that only attaches itself to [+human] nouns. We provide evidence to show that there are in fact two types of aa3-nominals. Their syntactic and semantic properties correlate with the number of syllables they consist of. There is a two-way split between disyllabic aa3-nominals and multisyllabic aa3-nominals. $A a_{3}$ in disyllabic $a a_{3}$-nominals should be treated as a prosodic template filler while $a a_{3}$ in multisyllabic aa3-nominals occupies a syntactic position in the referential layer of the Chinese nominal.

Keywords: proper names, phonological filler, dialectal grammar, Cantonese, familiarity

\section{Introduction}

This paper discusses the properties of Cantonese aa3 阿. It only appears with [+human] nouns, including common nouns, kinship terms and proper names. Some examples of these [+human] nouns are given in (1) and examples of them combining with $a a_{3}$ are given in (2). ${ }^{1}$

(1) a. Common nouns: siuzfaanz 'hawker', maai6jyu4louz 'fishmonger'

b. Kinship terms: saizlouz 'younger brother', muiz 'younger sister'

c. Proper names: ming $4 j a a_{1}$ 'Ming Jan', pitıtaa4 'Peter'

(2) ai. aa3-siu2faan 2 (ah-hawker $)^{2}$

aii. aa3-maai6jyu4louz (ah-fishmonger)

bi. aa3-saizlou2 (ah-younger brother)

bii. aa3-mui2 (ah-younger sister)

ci. aa3-ming4jan1 (ah-Ming Jan)

cii. aa3-pititaa $4 \quad(\text { ah-Peter })^{3}$

1. The Cantonese romanization scheme adopted here is Jyutping. 
Aa3-nominals are linguistically interesting in that their syntactic and semantic properties correlate with the number of syllables they consist of (Sio 2010). Specifically, there seems to be a two-way split between disyllabic aa3-nominals and multisyllabic aa3-nominals, with $a a_{3}$ included in the syllable count. ${ }^{4}$ To give an example, disyllabic aa3-nominals are compatible with classifiers, as in (3a), while multisyllabic aa3-nominals are not, (3b). The aa3-nominals are underlined in the following examples.

(3) a. ngo5 go3 aa3-mui2

1SG CL ah-younger sister

'my younger sister'

b. *ngo5 go3 aa3-sai3mui2

1SG CL ah-younger sister ${ }^{5}$

Intended reading: 'my younger sister'

In this paper, we discuss the similarities and differences between disyllabic aa3-nominals and multisyllabic aa3-nominals. We argue that their respective properties can be accounted for if we assume that $a a_{3}$ is always [+human] and [+familiar]. In disyllabic aa3-nominals, it is inserted as a prosodic filler for an iambic template that applies to monosyllabic [+human] 'familiar' terms (e.g. proper names, kinship terms and nicknames) as proposed by Yip (1992). The term 'familiar' here refers to social familiarity rather than discourse familiarity in the sense of Heim (1982). Disyllabic aa3-nominals can follow classifiers and can have a non-referential reading. Syntactically, we propose that disyllabic aa3-nominals are syntactic atoms (Di Sciullo \& Williams 1987) and are inserted in the head N. Whether they are interpreted referentially or not depends on whether they remain in the lexical layer or move to the referential layer of the nominal projection. Multisyllabic aa3-nominals, on the other hand, are always referential and can only be used designatively and vocatively. Unlike its template filler counterpart, we propose that $a a_{3}$ in multisyllabic aa3-nominals occupies the referential layer in the

2. Following the suggestion of an anonymous reviewer, we use $a h$ as the gloss of $a a_{3}$ as $a h$ is widely used in English-language novels as an informal Romanization for the Chinese character 阿 (e.g. ah Wong).

3. $A a_{3}$ is also compatible with loan proper names from English (Zhan 2002) or other languages. In actual usage, the pronunciation of these loans might be altered due to influence from Cantonese phonology. For instance, Peter is pronounced as pit1taa4 (in Jyutping) in spoken Cantonese, with the consonant geminated to function as the coda of the first syllable and the onset of the next. See Yip (1992:26) for discussion.

4. Multisyllabic aa3-nominals refer to nominals with 3 or more syllables.

5. In Example (3), mui2 and sai3mui2, as far as we can tell, do not differ in meaning. They both mean 'younger sister'. 
Chinese nominal, accounting for its obligatory referential reading. In the pool of Cantonese [+human] terms, only proper names and kinship terms can be used designatively and vocatively. Common nouns cannot be used in these two ways except for a few terms of professions such as jissang 1 'doctor' and lou si $_{1}$ 'teacher', which encode a closer and more permanent relationship with the speaker. We suggest that a [+familiar] feature enables a Cantonese [+human] noun to be used like a proper name. Kinship terms and common nouns like jissang1 'doctor' and lou5si1 'teacher' have a lexical [+familiar] feature and thus they can be used referentially and vocatively. When $a a_{3}$ is added to other [+human] common nouns, $a_{3}$ makes the whole noun phrase [+ familiar], enabling them to be used like proper names as well.

The paper is organized in the following manner. $\$ 2$ provides an overview of the distribution of aa3. $\S 3$ presents some previous analyses on aa3. In $\S 4$, we examine the grammatical differences between disyllabic aa3-nominals and multisyllabic aa3-nominals. In $\S 5$, we present our treatment for disyllabic $a a_{3}$-nominals. In $\S 6$, an analysis for multisyllabic aa3-nominals will be presented. We discuss some consequences of our analysis and tie up loose ends in $\S 7$.

\section{An overview}

The Cantonese $a a_{3}$ only combines with [+human] nouns, including common nouns, kinship terms, and proper names. An ungrammatical example of $a a_{3} \mathrm{com}$ bining with a [-human] common noun is given in (4c):

(4) Aa3 with common nouns:
a. aa3-siu2faan2
(ah-hawker)
b. aa3-maai6jyu4lou2 (ah-fishmonger)
c. ${ }^{*}$ aa3-syu1toi2 (ah-desk)

(5) Aa3 with kinship terms:
a. aa3-saizlou2 (ah-younger brother)
b. aa3-mui2 (ah-younger sister)

When $a_{3}$ appears with proper names, there are several possibilities. Aa3 could be used with the whole name (including the surname and the given name) as in (6a) (the surname is underlined). Aa3 could be used with only the given name, as in (6b). Aa3 could also be used with only the surname, as in (6c). Non-human proper names are not compatible with $a a_{3}$, as shown in (6d).

$A a_{3}$ with proper names:

(6) a. aa3-wong siurming $4^{2}$ 

b. aa3-siu2ming 4
c. aa3-wong2
d. *aa3-jat6bun2 'Japan'

Aa3 can also combine with titles, as in (7):

(7) aa3-dak6sau2

ah-Chief Executive of the Special Administrative Region

The only exception is personal pronouns. Even though they are [+human], they are not compatible with aaz:

(8) ${ }^{\star} a a_{3}-k e o i 5$

ah-3SG

In $\mathrm{Wu}$ dialects, such as Ningbo and Shanghainese, it is possible to have /a/ added to pronouns (Huang 1996:39).

In addition to common nouns, kinship terms and proper names, aa3 is also compatible with the question word binigo3 'who' (Matthews \& Yip 2011; Tang 2015: 59), which is also [+human]. Though when following aa3, as in (9), binigo3 'who' is no longer interpreted interrogatively. Instead, it has the reading of a specific person that the speaker knows but could not remember the name of. The following example is taken from Matthews \& Yip (2011:375): ${ }^{6}$

(9) Aa3-bin1go3 waa6 jiu3 ceng2 ngo5dei6 sik6 maan5faan6 wo5.

ah-who say want invite 1sG-PL eat dinner $\mathrm{SFP}^{7}$

'What's-his-name says s/he wants to invite us to dinner."

Aa3-binigoz in (9) does not function as a regular wh-word, but rather as a placeholder for the name of an individual.

$A a 3$ can only appear when attached to a [+human] noun. It cannot appear on its own and is often referred to as a prefix denoting familiarity (Matthews \& Yip 2011:43). There are two dimensions which are fundamental to the analysis of social relation (Brown \& Gilman 1960; Hudson 1996): the dimensions of power and solidarity. Terms of address often incorporate these two dimensions. The use of honorifics is a reflex of sensitivity to the dimension of power, an asymmetrical relation. The other dimension is solidarity, the feeling of unity or agreement, which is a symmetrical relation (Brown \& Gilman 1960). It concerns social dis-

6. The glosses and the romanization have been revised.

7. $\mathrm{SFP}=$ Sentence-final particle

8. Note that without $a a_{3}$, (9) will be ungrammatical. The sentence-final particle wo5 is not compatible with an interrogative reading that the wh-word binigoz would have in the absence of $a a_{3}$. 
tance between people, for instance, the use of the second person pronouns in French concerns both power and solidarity, e.g. vous vs. tu (Hudson 1996). In terms of pragmatic functions, $a a_{3}$ can be viewed as a solidarity marker, which can be used both upward, downward, or with equals. $A a_{3}$ indicates solidarity between the speaker and the referent of the post-aa3 [+human] noun.

\section{Superior and solidarity:}

(10) a3-lousbaan2

ah-boss

\section{Equality and solidarity:}

(11) aa3-ming 4 (in addressing a colleague called Ming)

ah-Ming

\section{Inferiority and solidarity:}

(12) aa3-saizlou2

ah-younger brother

Chao (1968:216) mentions the use of $a h$ - in Mandarin, stating that it is a prefix and its occurrence is rare both lexically and textually. Chao mentions that the only word in Mandarin with this prefix is the term of address ah ge [ah-older brother], used as a title at the court of the Manchu Dynasty. Another case we find which uses the $a h$-marker in Mandarin is àyi 阿姨, a term used to refer to one's maternal aunts or working-age women.

Huang (1996:39) mentions that the use of the prefix ah- has slowly and gradually become obsolete in Northern dialects since the beginning of the Song Dynasty. Similarly, Chao also notes that the occurrence of $a h$-in contemporary Mandarin is limited to forms borrowed from dialects. Indeed, a lot of Chinese dialects (e.g. Wu, Min, Hakka) have a counterpart of $a a_{3}$ (/a/ and its cognates, Huang 1996:38-39), an element that is placed immediately before terms of address such as kinship terms and proper names. In Cantonese, it is also possible to place $a a_{3}$ in front of a number (Yip 1992:29, Bauer \& Benedict 1997: 205). The number represents the birth order of a person and the term [aa3-number] is then used as an address term for that person like a nickname. For example, [aa3-saam1 'three'] can be used to refer to the third child. When there are only two siblings, it is also possible to use [aa3-daai6 'big'] to refer to the older one and [aa3-sai3 'small'] to refer to the younger one. This usage, with (/a/ or its cognates) preceding a number/adjective that reflects birth order, is also available in $\mathrm{Wu}, \mathrm{Min}$, and Hakka, as reported in Huang (1996:38-39). Yip (1992: 29) also mentions the use of $a a_{3}$ with adjectives (other than 'big' and 'small'). For example, [aa3-fei2 'fat'] can be used as a nickname for someone who is fat. In Hong Kong, there is an actor 
called Can 4 Baak 3 Coeng 4 with the stage name [aa3-lek1 'smart']. ${ }^{9}$ The stage name goes with his stage persona which is both smart and confident.

\section{Previous analyses}

Lyons (1999:153) refers to $a a_{3}$ as a vocative marker in Cantonese. We believe this captures an important insight of $a a_{3}$, as all aa3-nominals (both disyllabic and multisyllabic) can be used vocatively. This, however, cannot be the function of $a a_{3}$ as aa3-nominals can also be used designatively as shown in (13).

\section{(13) Aa3-ji1sang1/sai3lou2/siu2ming4 heoi3-zo2 bin1 ne1? \\ Ah-doctor/younger brother/Siu Ming go-ASP where $\mathrm{QP}^{10}$ \\ '(lit.) Where did doctor/younger brother/Siu Ming go?'}

Furthermore, disyllabic aa3-nominals can also be used non-referentially, a usage which will be discussed in $\S 4$. In other words, aa3-nominals are not restricted to vocative contexts, and thus $a a_{3}$ cannot be a vocative marker.

Yip (1992) claims that $a a_{3}$ is a prefix inserted to satisfy a disyllabic iambic prosodic template when a familiar human term is monosyllabic: ${ }^{11}$

(14) $\sigma\left[+\right.$ familiar] [+human] $\rightarrow a a_{3} \sigma[+$ familiar] [+human]

(15) a. Surnames:

jip6 $\rightarrow$ aa3-jip2

b. Family relations:

mui2 'younger sister' $\rightarrow$ aa3-muiz

c. Names based on birth order:

$j i 6^{\text {'two' } \rightarrow a a_{3}-j i 2}$

d. Nicknames:

fei4 'fat' $\rightarrow$ aaj-fei2

Yip (1992) provides a few motivations for treating $a a_{3}$ as a filler element. Phonologically, she argues that an epenthetic syllable is supposed to use unmarked featural material. Following Cheng (1990), Yip (1992) argues that $a a_{3}$ is unmarked

9. We would like to thank an anonymous reviewer for providing us with this example.

10. $\mathrm{QP}=$ Question particle

11. In addition to the insertion of $a a_{3}$, the second syllable is sometimes made more prominent by the addition of high tone and it is somewhat lengthened as in (15a) (Chao 1947; Yip 1992: 28). The result is that tones 1 (high flat) and 2 (high rising) remain unchanged, while all other tones change to tone 2, which ends high. See also Bauer \& Benedict (1997:202) for a detailed discussion of changed tone (or pinjam) in Cantonese surnames and nicknames. 
because /a/ and /a:/ are the only vowels that are not subject to any co-occurrence restrictions with particular onsets and codas, suggesting that they lack most or all place features. Yip (1992) also remarks that aas has a mid tone, 33 (tone 3 in Jyutping), which is likely to be the default tone (see also Sybesma \& Li 2007).

To fulfill the iambic template for monosyllabic familiar human terms, the addition of an extra syllable can also be achieved by reduplication. ${ }^{12}$ Proper names and kinship terms often undergo reduplication to form an iambic foot while common nouns generally do not (except in 'baby-talk'). In the case of proper names, reduplication only applies to given names. The resulting nominals feel informal and intimate. Some examples are given below:

(16) a. Given names:

ming $4 \rightarrow$ ming 4 ming 2

b. Kinship terms:

baa1 'father' $\rightarrow$ baa4-baa1

maa1 'mother' $\rightarrow$ maa4-maa1

Yip (1992) does not discuss the use of $a a_{3}$ in polysyllabic aa3-nominals, though she did mention that as a possibility in Footnote 24. Obviously in multisyllabic aa3-nominals, aa3 cannot be a disyllabic iambic template filler.

\section{Disyllabic $a a_{3}$-nominals vs. multisyllabic $a a_{3}$-nominals}

In this section, we discuss the syntactic and semantic differences between disyllabic aa3-nominals and multisyllabic aa3-nominals. These differences include compatibility with classifiers, ability to be non-referential as well as possessor reference possibilities.

\subsection{Compatibility with classifiers}

As mentioned in the introductory section, when $a a_{3}$ is preceded by a classifier, the [+human] term following $a a_{3}$ has to be monosyllabic. When the classifier is not immediately followed by $a a_{3}$, nouns of any length can be in the post-classifier position:

a. ngo5 go3 aa3-go1

1SG CL ah-older brother

'my older brother'

12. Yet another way to satisfy the template is to add lous 'old' in front of a surname, as in lous can 2 'old Chan'. 


\section{b. * ${ }^{\star} g o 5$ go3 aa3-go4-go1}

1SG CL ah-older brother

Intended reading: 'my older brother'

c. ngo5 go3 daai6 biu2ze2

1SG CL big older female cousin

'my big older female cousin'

Disyllabic aa3-nominals can appear in a post-classifier position while multisyllabic aa3-nominals cannot.

\subsection{Ability to be non-referential}

Disyllabic aa3-nominals can have a non-referential interpretation. They can function as nominal predicates while multisyllabic aa3-nominals cannot. Multisyllabic aa3-nominals are always referential (Sio 2010). Examples in (18) illustrate this using the kinship term 'younger sister', which can appear as a disyllabic aa3-nominal aa3-muiz, a multisyllabic aa3-nominal aa3-saizmuiz or a bare kinship term saizmuiz. The bare kinship term here patterns with the disyllabic aa3-nominal:

(18) a. Ngo5 dong3 keoi5 aa3-mui2 gam2 gaa3.

$1 \mathrm{SG}$ treat $3 \mathrm{SG}$ ah-younger sister like-that SFP

'I treat her like a younger sister.'

b. *Ngo5 dong3 keoi5 aa3-sai3mui2 gam2 gaa3.

$1 \mathrm{SG}$ treat $3 \mathrm{SG}$ ah-younger sister like-that SFP

Intended reading: 'I treat her like a younger sister.'

c. Ngo5 dong3 keoi5 sai3mui2 gam2 gaa3.

$1 \mathrm{SG}$ treat $3 \mathrm{sg}$ younger sister like-that SFP

'I treat her like a younger sister.'

Dong3...gam2 'treat...like' forces an intentional reading of 'younger sister'. The ungrammaticality of (18b) stems from aa3-saiz-muiz not being able to have a nonreferential reading.

Table 1. Interpretation of $a a_{3}$ with kinship terms

\begin{tabular}{lll}
\hline Form & & Non-referential? \\
\hline (a) $a a_{3}$-mui2 & {$\left[a a_{3} \sigma\right]$} & YES \\
(b) $a a_{3}$-sai3mui2 & {$\left[a a_{3} \sigma \sigma \ldots\right]$} & NO \\
(c) sai3mui2 & {$[$ bare kinship term $]$} & YES \\
\hline
\end{tabular}




\subsection{Possessor reference possibility}

The three forms of 'younger sister' in Table 1 also show variation in possessor reference (Sio 2010). This is illustrated in (19):
(19)
a. Pit1taa4 joek3-zo2
aa3-mui2
heoi3 sik6-faan6.

Peter make appointment-ASP ah-younger sister go eat-rice

'Peter is meeting his younger sister/the speaker's younger sister for a meal.'

b. Pit1taa4 joek3-zo2 aa3-sai3mui2 heoi3 sik6-faan6.

Peter make appointment-AsP ah-younger sister go eat-rice

'Peter is meeting the speaker's younger sister for a meal.'

c. Pit1taa4 joek3-zo2 sai3mui2 heoi3 sik6-faan6.

Peter make appointment-ASP younger sister go eat-rice

'Peter is meeting his younger sister/the speaker's younger sister for a meal.'

The disyllabic aa3-muiz in (19a) can take the subject or the speaker as its possessor. This is the same as the bare kinship term saizmuiz in (19c). The multisyllabic aa3-saizmuiz in (19b) can only be interpreted as the speaker's younger sister. The three forms are not identical.

Table 2. Possessor reference variation

\begin{tabular}{ll}
\hline Form & Possessor reference possibilities \\
\hline (a) $a a_{3}-$ mui2 $\left[a a_{3} \sigma\right]$ & the subject's younger sister or the speaker's younger sister \\
(b) aa3-saizmui2 $\left[a a_{3} \sigma \sigma . ..\right]$ & the speaker's younger sister \\
(c) sai3mui2 $[$ bare kinship term] & the subject's younger sister or the speaker's younger sister \\
\hline
\end{tabular}

The above data show that disyllabic aa3-kinship terms behave like bare kinship terms in that they can have flexibility in possessor reference while multisyllabic aa3-kinship terms are totally inflexible. They are always interpreted as related to the speaker (can only be the speaker's younger sister in this case).

Again, this shows disyllabic aa3-nominals pattern with bare nouns while multisyllabic aa3- nominals have different properties.

\section{Disyllabic aa3-nominals}

The contrast in properties between disyllabic aa3-nominals and multisyllabic aa3-nominals unveils a correlation between prosody and grammatical properties. Similar observations have been made in Mandarin by Feng (2009), though based on different constructions. Feng (2009) discusses several syntactic constructions like [verb +object] (e.g. fu-ze vs. fu-zeren 'carry-responsibility'), [auxiliary + verb] 
(ke-pa vs. keyi-pa 'can-terrify'), [verb + resultative] (e.g. shuo-ming vs. shuomingque 'say-clear') and [adjective + noun] (da xiongmao 'big panda' vs. da-yan 'big-goose'), and observes that disyllabic constructions have different syntactic properties from trisyllabic constructions. Specifically, only disyllabic ones exhibit lexical properties. Feng (2009) concludes that disyllabic forms constitute minimal prosodic units in Chinese and thus could be interpreted as compound words by native speakers while trisyllabic forms cannot. As pointed out by Feng (2009), it is unclear, however, what the syntactic structure should look like as these minimal prosodic units may also circumscribe certain syntactic phrases.

Following Yip (1992), we assume that $a a_{3}$ in disyllabic $a a_{3}$-nominals are template fillers, but with semantic features. We would like to propose that $a a_{3}$ is [+human] and [+familiar]. Since it is monosyllabic, [+human] and [+familiar], it is the ideal filler for the iambic template for familiar human terms.

The next question is the syntactic status of disyllabic aa3-nominals. When disyllabic aa3-nominals appear after the classifier, they can be immediately preceded by bare modifiers as well as modifiers with the marker $g e 3:{ }^{13}$

(20) ngo5 go3 leng3 neoi2 aa3 mui2

1SG CL beautiful female ah younger sister

'my beautiful younger sister'

(21) ngo5 go3 leng3 neoi2 ge3 aa3 mui2

1SG CL beautiful female GE ah younger sister

'my beautiful younger sister'

As noted in Sio (2006), bare modifiers and modifiers with the marker exhibit different properties, among others, bare modifiers are always closer to the noun than modifiers with the marker:

(22) ngo5 go3 paa3 cau2 ge3 leng3 neoi2 aa3 mui2

1SG CL shy GE beautiful female ah younger sister

'my shy beautiful younger sister'

(23) *ngo5 go3 leng3 neoi2 paa3 cau2 ge 3 aa 3 mui2

1SG CL beautiful female shy GE ah younger sister

'my shy beautiful younger sister'

For (23) to be grammatical, there needs to be a pause after leng3 neoiz, indicating the structure is not the same.

Following Sio (2006), we assume that bare modifiers are in the SpecNP, with the option of allowing multiple Specs while modifiers with the marker are

13. Ge3 嘅 is the equivalent of the modification marker $d e$ 的 in Mandarin. 
adjuncts to NP. Disyllabic aa3-nominals are $\mathrm{N}$ heads. They are inserted in N syntactically, just like bare nouns. They can be used as predicates and can follow classifier, if present (as discussed in $§ 4.1$ )

(24)

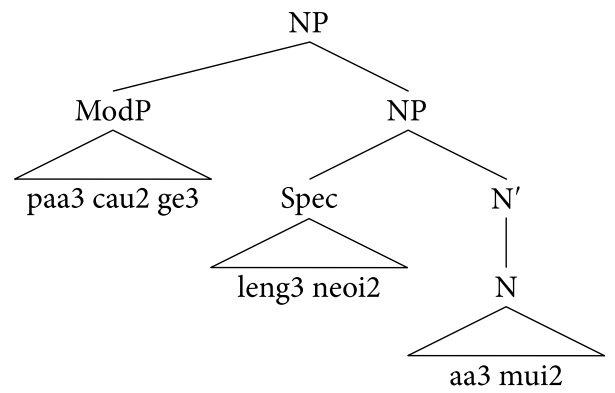

In addition to the non-referential usage, disyllabic aa3-nominals can also function like proper names in the sense that they can be used vocatively and designatively. We assume that in such cases, there is movement of the disyllabic aa3-nominal from the NP layer to the DP layer (Longobardi 1994). ${ }^{14}$ One possibility is for the whole NP to move to SpecDP.

Yip (1992) claims that the template filler $a a_{3}$ is only used with [+human] familiar terms. Indeed, when $a a_{3}$ is combined with monosyllabic [+human] common nouns that are unlikely to be interpreted as familiar, the resulting nominals do not sound natural.

(25) ??aa3-caak2

ah-thief

(26) ??aa3-gun

ah-official

An anonymous review suggested that (25) can be used under a strong context where the thief is somehow made familiar. We shall address these special cases in $\S 7$.

A special type of disyllabic aa3-nominals we would like to discuss are nouns that are literally kinship terms, but are so bleached that they can also be used as common nouns. Consider the following disyllabic aa3-nominals: ${ }^{15}$

14. We understand that the nature of the nominal referential layer in Chinese is a contentious issue. We follow works that argue for a referential layer in Chinese (see Cheng \& Sybesma 1999; Sio 2006, among others). The exact nature of such a layer is beyond the scope of this paper. We use the term DP as a general term for a referential layer.

15. We would like to thank an anonymous reviewer for pointing out that in addition to suk1 'uncle' and sam2 'aunt', the meanings of ze1 'older sister' and go1 'older brother' have also been bleached. [Aa3-ze1] and [aa3-go1] can nowadays be used to refer to early middle-aged men and women (a bit younger than [aa3-suk1] and [aa3-sam2]). 
(27) $a a_{3}-s u k_{1}$

ah-uncle

'middle-aged man/uncle'

(28) aa3-sam2

ah-aunt

'middle-aged woman/aunt'

Literally, suk1 'uncle' refers to the younger brothers of one's father and sam2 'aunt' refers to the wives of one's father's male siblings. These readings are still available. Additionally, these terms are bleached to the extent that nowadays they can also have a common noun reading: they refer to, respectively, middle-aged men and middle-aged women. Thus, both (27) and (28) are ambiguous. Both terms can have a kinship term reading as well as a common noun reading.

Regular kinship terms that are not common nouns cannot appear in existential constructions. Kinship terms encode a two-place relationship. Thus, they can only be interpreted referentially if a possessor is provided, be it overt or covert. The need for a possessor explains why kinship terms cannot appear in existential sentences, unlike common nouns. This is illustrated in (29):

a. *Fong2-jap6min6 jau5 saam1 go3 sai3mui2.

room-inside have three $\mathrm{CL}$ younger sister

Intended reading: 'There are three younger sisters in the room.'

b. Fong2-jap6min6 jau5 saam1 go3 sau6fo3jyun4.

room-inside have three CL salesperson

'There are three salespersons in the room.'

Some kinship terms have developed semantic characteristics that are independent of their relation, such as maa1mi4 'mother', which can be interpreted as a common noun (a female who has children), and they can then appear in contexts where only common nouns are allowed:

(30) Fong2-jap6min6 jau5 saam1 go3 maa1mi4.

room-inside have three CL mother

'There are three mothers in the room.' (e.g. in the context of a maternity ward)

As expected, aa3-suk1 and aa3-sam2 can appear in existential constructions but bearing a common noun reading only:

(31) Fong2-jap6min6 jau5 saam1 go3 aa3-suk1/aa3-sam2.

room-inside have three $\mathrm{CL}$ ah-uncle/ah-aunt

'There are three middle-aged men/middle-aged women in the room.' 
In the existential sentence in (31), the kinship term reading is not available as there is no possessor. In (32) below, both a kinship term reading and a common noun reading are available.

(32) Aa3-suk1, mai5-haang4-zyu6!

ah-uncle NEG-go-yet

a. '(lit.) Middle-aged man, don't go yet!'

(common noun reading)

b. '(lit.) Uncle, don't go yet.'

(kinship term reading)

In (33), only a kinship term reading is possible. When a classifier is used, only a common noun reading is possible, as in (34):

(33) Ngo5 kam4-jat6 gin3-dou2 aa3-suk1 aa3.

1SG yesterday see-ASP ah-uncle SFP

'I saw (my) uncle yesterday.'

(kinship term reading only)

(34) Ngo5 kam4-jat6 gin3-dou2 go3 aa3-suk aa3.

1 SG yesterday see-ASP CL ah-uncle SFP

'I saw the middle-aged man yesterday.' (common noun reading only)

\section{Multisyllabic aa3-nominals}

\subsection{Not just definite}

Multisyllabic aa3-nominals are always referential, and they must be used either vocatively or designatively, like proper names.

Generally, Chinese only allows definite subjects (see Chao 1968; Cheng \& Sybesma 1999, among others). Bare common nouns in Cantonese cannot have a definite reading (Cheng \& Sybesma 1999; Sio 2006; Tang 2011) and thus are banned from the subject position (except for a generic reading). This is shown in (35a). A vocative usage also sounds deviant, as illustrated in (35b).

a. *sau6fo3jyun 4 ci4-dou3.

salesperson late

Intended reading: 'The sales person was late.'

b. ?? sau6fo6jyun4, ngo5 soeng5 maai5 nei1 go3. salesperson 1SG want buy this CL Intended reading: 'Sales person, I want to buy this one.'

As discussed in Sio (2010), when $a a_{3}$ is added to a [+human] common noun, the situation is flipped. The resulting expression can then be used designatively and vocatively. This is illustrated in (36a) and (36b) respectively: 
(36) a. Aa3-sau6fo3jyun4 ci4-dou3.

Ah-salesperson late

'Salesperson is late.'

b. Aa3-sau6fo3jyun4, ngo5 soeng2 maai5 nei1 go3.

Ah-salesperson 1sG want buy this CL

'Salesperson, I want to buy this one.'

The grammaticality of ( $36 \mathrm{a}$ ) shows that aa3 makes the resulting expression definite. Sio (2010) points out that $a a_{3}$ in fact does more than that. She notes that in (36a) and (36b), the interpretation of [aa3-salesperson] is not 'the salesperson', but rather something like a proper name. To see the difference, consider the situation set up in (37) with (38a) as a follow-up sentence. In such a situation, even though the hearer probably cannot identify the salesperson, a definite noun phrase can still be licensed by uniqueness in association with the context (Lyons 1999: 8). As for Cantonese, among the two potential follow-up sentences, only (38b), but not $(38 \mathrm{c})$, would be a natural follow-up sentence.

(37) I went to the new corner shop yesterday...

(38) a. The salesperson was very pretty.

b. Go3 sau6fo3jyun4 hou2 leng3 gaa3.

CL salesperson very pretty SFP

c. "Aa3-sau6fong3jyun4 hou2 leng3 gaa3.

Ah -salesperson very pretty SFP

[Cl-N] phrases in Cantonese can be interpreted as definite (Cheng \& Sybesma 1999). [Cl salesperson] in (38b) is interpreted as 'the salesperson' in the corner shop mentioned in (37). [Aa3-sau6fong3jyun4 'salesperson'] in (38c), however, is interpreted as a unique individual that both the hearer and speaker have in mind and the reference of which is not dependent on the previously introduced corner shop. In other words, it is something akin to a proper name (Sio 2010).

Consider the following examples:

(39) My best friend is John.

(Geurt 1997:323, (10))

(40) Who's John?

Geurt (1997) uses (39) to show that proper names can be used when the referent is new to the addressee. Abbott (2002) argues that (39) invites the question (40), thus arguing that unfamiliar proper names have to be introduced into the discourse before being used. The use of proper names generally supposes that the name is known to the hearer (Prince 1992). This explains why (38c) is deviant as a follow-up sentence for (37). The proper name-like [aa3-salesperson] has not 
been introduced to the discourse previously. (38b) is a regular definite description and can be licensed by uniqueness in association with the context (Lyons 1999: 8). [Aa3-salesperson] in (38c), being proper name-like, cannot be licensed this way. Its proper name-like nature, on the other hand, licenses its vocative usage in (36b). To provide yet another minimal pair between a definite reading and a proper name-like reading, imagine a situation where you and a friend are shopping in a mall. A salesperson has been helping you, and when you want to pay, you could not find him anymore, then it is natural for one of you to utter the following:

(41) Aa3-sau6fo3jyun4 heoi3-zo2 bin1 aa3?

ah salesperson go-ASP where SFP

'Where does Salesperson go?'

(42) Go3 sau6fo3jyun4 heoi3-zo2 bin1 aa3?

CL salesperson go-ASP where SFP

'Where did the salesperson go?'

Both (41) and (42) are pragmatically natural in this context. (42) feels neutral. (41), however, feels more intimate, as if the salesperson is a friend and you are calling him by his name (but you don't know his name, so you make one up using his job title).

To sum up, multisyllabic aa3-nominals are not just definite. They are like proper names with intimacy.

\subsection{Resistance to modification}

Even though polysyllabic aa3-nominals behave like proper names, they are different from proper names in one respect: they cannot be modified by modifiers appearing to the left of $a a_{3}$ (Sio 2010).

As shown earlier on, disyllabic aa3-nominals can be modified, as in (43). Common nouns, kinship terms and proper names can be modified, (44). When $a_{a}$ is added to these nouns to form multisyllabic aa3-nominals, the resulting nominal can no longer be modified. This is shown in (45).

(43) a. hou2 gou1 ge3 aa3-ming4

very tall GE ah-Ming

'(lit.) very tall ah-Ming'

b. hou 2 gou1 ge3 aa3-mui2

very tall $\mathrm{GE}$ ah-younger sister

'(lit.) very tall younger sister' 
(44) a. hou2 gou1 ge3 sau6fo3jyun4

very tall GE salesperson

'tall sales person(s)'

b. hou 2 gou 1 ge 3 sai3lou2

very tall GE younger brother

'tall younger brother'

c. hou 2 goul ge3 Ming4 Jan1

very tall GE Ming Jan

'tall Ming Jan'

(45) a. *hou2 gou1 ge3 aa3-sau6fo3jyun4

very tall GE ah-sales person

Intended reading: 'tall sales person'

b. *hou 2 gou 1 ge3 aa3-sai3lou 2

very tall GE ah-younger brother

Intended reading: 'tall younger brother'

c. *hou2 gou1 ge3 aa3-Ming4 Jan1

very tall GE ah-Ming Jan

Intended reading: 'tall Ming Jan'

As mentioned in $\S 5$, there are two types of modifiers, one with the marker and the other type bare. Bare modifiers are also not allowed to appear to the left of aa3. If $g e 3$ is absent in the examples in (45), the phrases are still ungrammatical. In polysyllabic aaz-nominals, aaz has to be on the left edge.

It is however possible, though mildly degraded, to have modifiers appearing between $a a_{3}$ and the noun in multisyllabic aa3-nominals:

(46) aa3-paa3cau2 sau6fo3jyun4

ah-shy salesperson

(47) aa3-paa3cau2 ge3 sau6fo3jyun4

ah-shy GE salesperson

This is not possible for disyllabic aa3-nominals:

(48) *aa3 paa3cau2 (ge3) mui2

Ah shy GE younger sister

\subsection{Analysis}

Multisyllabic aa3-nominals are always referential. We propose that aas in in the DP layer. In particular, we propose that $a a_{3}$ is in SpecDP, the same position as possessors in Cantonese (SpecSP, Specificity Phrase, in Sio 2006). This is moti- 
vated by the observations that $a a_{3}$ has very similar distribution as possessors. We present the arguments below.

Unlike kinship terms, multisyllabic aa3-nominals also cannot take possessors:

(49) a. ngo5 sai3mui2

$1 \mathrm{SG}$ younger sister

'my younger sister'

b. *ngo5 aa3-sai3mui2

1SG ah-younger sister

Intended reading: 'my younger sister'

If both $a a_{3}$ and possessors occupy the specifier position of the DP layer, it explains why they are in complementary distribution.

$\mathrm{Aa}_{3}$ and possessors also behave similarly in that they seem to close off the DP domain. Nothing can appear to the left of it:
a. *hou2 goul ge3 ngo5 sai3mui2
very tall GE 1 sG younger sister
Intended reading: 'my very tall younger sister'
b. *hou gou1 ge3 aa3 sai3mui2
very tall GE ah younger sister
Intended reading: 'my very tall younger sister.'

This also provides a way of explaining the possession facts in (19), repeated here in (51):

a. Pit1taa4 joek3-zo2

aa3-mui2

heoi3 sik6-faan6.

Peter make appointment-AsP ah-younger sister go eat-rice 'Peter is meeting his younger sister/the speaker's younger sister for a meal.'

b. Pit1taa4 joek3-zo2 aa3-sai3mui2 heoi3 sik6-faan6.

Peter make appointment-ASP ah-younger sister go eat-rice 'Peter is meeting the speaker's younger sister for a meal.'
c. Pit1taa4 joek3-zo2
sai3mui2
heoi3 sik6-faan6.

Peter make appointment-ASP younger sister go eat-rice

'Peter is meeting his younger sister/the speaker's younger sister for a meal.'

To recap the interpretations, in (51a) and (51c), without an overt possessor, the disyllabic aa3-nominal aa3-muiz and the bare kinship term saizmuiz can be interpreted as either Peter's younger sister or the speaker's younger sister. In (51b), aa3-saizmuiz can only be interpreted as the speaker's younger sister. In aa3-mui2 and sai4muiz, the possessor variable, which is in SpecDP, can be bound by the subject or contextually bound by the speaker. In aa3-saizmuiz, aa 3 takes the position of the variable. The relational reading, that it is the speaker's younger sister, is 
derived by pragmatic means. The reference domain for proper names is by default speaker-determined. Imagine A is having a conversation with B. A's sister's name is Lisa. When A mentions Lisa, it is natural for B to interpret referent as A's sister rather than some other Lisa. This is because among all the Lisas they potentially know, this Lisa happens to be the most prominent in the conversational context. This can of course be overridden, given the appropriate context.

In view of the above, we propose the following structure for multisyllabic aa3-nominals:

$(52)$

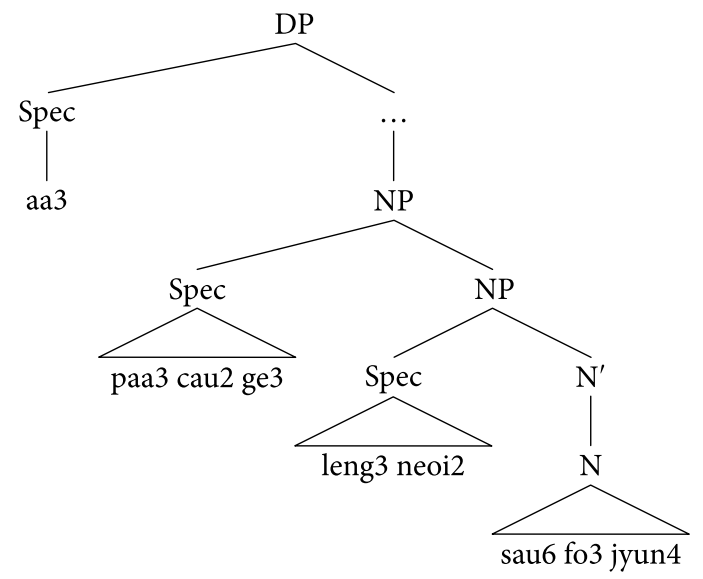

Multisyllabic aa3-nominals are interpreted like proper names. The properties of proper names are such that they are rigidly designating and can be used vocatively and designatively. In Cantonese, as mentioned before, two other types of noun phrases can do that: kinship terms, and certain terms of professions like jiisang1 'doctor' and loussi1 'teacher'.

a. Sai3lou2/ji1sang1/lou5si1 zou2-san4!

Younger brother/doctor/teacher good morning

'Good morning younger brother/doctor/teacher!'

b. Sai3lou2/ji1sang1/lou5si1 dim2 gong2 aa3?

Younger brother/doctor/teacher how say SFP?

'(lit.)What did younger brother/doctor/teacher say?'

These are professions that encode a closer and more permanent relationship with the speaker. Other professions like siu2faan2 'hawker' or sau6fo3jyun4 'salesperson' would sound strange in (53). However, once you add with $a a_{3}$ to these regular [+human] common nouns, they can. 
(54) a. Aa3-sau6fo3jyun4/ aa3-siu2faan2 zou2-san4!

Ah-salesperson ah-hawker good morning

'Good morning salesperson/hawker!'

b. Aa3-sau6fo3jyun4/aa3-siu2faan2 dim2 gong2 aa3?

Ah-salesperson how say SFP?

'What did salesperson/hawker say?'

The similarity between kinship terms and these special terms of professions (jiisang1 'doctor' and lou5si1 'teacher') can be explained if we assume that they both contain a [+familiar] feature and all Cantonese nouns with a [+familiar] feature can be used like proper names. Kinship terms are [+familiar] because they encode family relations. Nouns like jiisang1 'doctor' and lou 5 si $_{1}$ 'teacher' are [+familiar] because they encode a closer and more permanent relationship with an individual in the context of the profession compared with other professions such as 'fireman' or 'baker' for instance. ${ }^{16}$ If any noun that has [+familiar] feature can be used like proper names, this would account for why kinship terms and certain terms of professions can be used designatively and vocatively. When $a a_{3}$ is added to a non-familiar [+human] noun, e.g. sau6fo3jyun 4 'salesperson', aa3 makes the resulting noun phrase [+familiar], and this add-on [+familiar] feature enables them to be used like proper names as well.

Multisyllabic aa3-nominals are not compatible with classifiers. It is unclear to us how this can be derived syntactically. Given that proper names are also not compatible with classifier (except when they have a common noun reading), it could be that it is a semantic issue. We shall leave this issue for future research. ${ }^{17}$

16. Kinship terms and terms like jissang1 'doctor' and loussi1 'teacher' also pattern in the same way in possessives. While common nouns obligatorily require the presence a classifier in possessive constructions, these terms do not (see Sio 2003 for discussion):

(i) ngo5 (go3) mui4mui2/lou5si1

$1 \mathrm{sG} \quad$ CL $\quad$ younger sister/teacher

'my younger sister/teacher'

(ii) $n g o 5^{\star}($ go3 $)$ caang2

1SG CL orange

'my orange'

17. Sio (2010) proposes that the Classifier Phrase (ClP) is missing in multisyllabic aa3nominals. She assumes that modifiers with the marker gez are merged at the ClP level and thus explaining their incompatibility with classifiers and modification. This is problematic as modifiers with the marker ge 3 are allowed to appear to the right of $a a_{3}$ in multisyllabic aa3-nominals (though mildly degraded). One possibility is that when modifiers appear between $a_{3} 3$ and the noun phrase, the resulting nominals are not multisyllabic aa3-nominals. We did not pursue this line of reasoning, as we could find no evidence to support this. 


\section{Some consequences and loose ends}

In this paper, we have provided an overview on the differences between two types of aa3-nominals, disyllabic aa3-nominals and multisyllabic aa3-nominals, the differences are presented in the following table:

Table 3. A summary of differences between disyllabic aa3-nominals and multisyllabic aa3-nominals

\begin{tabular}{lllll}
\hline & Referentiality & $\begin{array}{l}\text { Compatibility } \\
\text { with classifiers }\end{array}$ & $\begin{array}{l}\text { Modification to } \\
\text { the left of } a a_{3}\end{array}$ & $\begin{array}{l}\text { Modification to } \\
\text { the right of } a a_{3}\end{array}$ \\
\hline $\begin{array}{l}\text { Disyllabic } \\
\begin{array}{l}a a_{3} \text {-nominals } \\
\text { (lexical) }\end{array}\end{array}$ & $\begin{array}{l}\text { Referential/ } \\
\text { non-referential }\end{array}$ & Yes & Yes & No \\
$\begin{array}{l}\text { Multisyllabic } \\
\begin{array}{l}a a_{3} \text {-nominals } \\
\text { phrasal) }\end{array}\end{array}$ & Always referential & No & No & Yes \\
\hline
\end{tabular}

The differences between disyllabic aa3-nominals and multisyllabic aa3-nominals can be partially accounted for if we adopt the following structures:

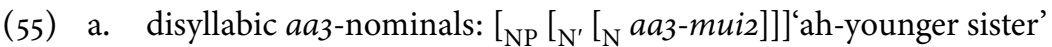

b. multisyllabic aa3-nominals: ${ }_{\mathrm{DP}} a a_{3}\left[{ }_{\mathrm{D}^{\prime}}\left[\ldots\left[_{\mathrm{NP}}\left[{ }_{\mathrm{N}^{\prime}}\left[{ }_{\mathrm{N}}\right.\right.\right.\right.\right.$ sau6fo3jyun 4$\left.\left.\left.\left.]\right]\right]\right]\right]$ 'ah-salesperson'

In all $a a_{3}$-nominals, $a a_{3}$ is [+human] and [+familiar].

Following Yip (1992), we assume that aa3 in disyllabic aa3-nominals is an iambic template filler for monosyllabic familiar human terms. Disyllabic aa3-nominals are syntactic atoms inserted in $\mathrm{N}$. This explains why disyllabic $a a_{3}$-nominals are compatible with classifiers and can be non-referential. When aa3 appears with (i) non-familiar [+human] terms or (ii) non-monosyllabic [+familiar] and [+human] terms, in both cases, the iambic template would not apply. In multisyllabic aa3-nominals, aa3 makes non-familiar [+human] term [+familiar]. The obligatorily referential reading for multisyllabic aa3-nominals comes from the syntactic position of $a a_{3}$. We propose that it is located in a specifier of the DP layer in Cantonese, capturing the similarities between aa 3 and possessors. This also makes multisyllabic aa3-nominals always referential. The [+familiar] feature enables these nominals to behave like proper names and just like proper names when they are interpreted referentially, multisyllabic aa3-nominals are not compatible with classifiers.

One reviewer raised the following interesting point. Most surnames in Chinese are monosyllabic and that calls for the insertion of $a a_{3}$ to form an iambic 
template. However, there are also a few surnames that are disyllabic, such as Au1joeng2. The analysis proposed here will treat $a a_{3}$ in $a a_{3}$-Can 2 differently from $a_{3} a_{3}$ in $a_{3}$-Auijoeng 2 even though they are both surnames. This is indeed the consequence of the analysis proposed here. However, we do observe a contrast between the two surnames:
a. hou2 gou1 ge3 aa3-Can2 very tall GE ah-Chan
b. *hou2 gou1 ge3 aa3-Au1joeng2 very tall GE ah-Auyeung

(57a) sounds natural while (57b) sounds bad. This shows that $a a_{3}$-Can2 and aa3-Auijoeng2 should indeed be treated differently, supporting Feng's (2009) idea that native speaker's intuition about the notion of a word is affected by the prosodic notion of Minimal Word (disyllabic forms), and it has consequences on syntax.

Yip's (1992) disyllabic template only applies to [+familiar] human nouns. Nonfamiliar monosyllabic human nouns, such as caak2 'thief' or gun1 'official', are not subject to the template requirement. An anonymous reviewer suggested that in a context where the familiarity of the thief is established, [aa3-caak2 'thief'] would then be acceptable. We concur with the reviewer's comment, but it would require a special context (e.g. your friend's job is a thief and your mother refers to him as aa3-caak2). Another anonymous reviewer points out that one can say [aa3-'sir'], which is a very common way to refer to a teacher or a police officer. When it is used to refer to a teacher, it is a [+familiar] noun. It is also a friendly way to refer to a policeman. Even though both can be used (given a special context for the former), there is one big difference between [aa3-caak2 'thief'] and [aa3-'sir']. [Aa3-'sir'] functions as a synonym for 'teacher' and 'policeman' and shares similar syntactic properties with the common noun 'teacher' and 'policeman'. For instance, it is compatible with classifiers. [Aa3-caak2 'thief'] is not compatible with classifiers, and if it is used, it is either used designatively or vocatively, identical to multisyllabic aa3-nominals.

(58) Fong2-jap6min6 jau5 saam1 go3 aa3-sir.

room-inside have three CL ah-policeman/teacher

'There are three policemen/teachers in the room.'

(59) ${ }^{\star}$ Fong2-jap6min6 jau5 saam1 go3 aa3-caak2.

room-inside have three CL ah-thief

'There are three thieves in the room.'

In view of our proposed analysis, [aa3-'sir'] is a syntactic atom. Since the template filler $a a_{3}$ is only added to [+human] [+familiar] terms, we are forced to conclude 
that 'sir' is lexically [+familiar]. In [aa3-caak2 'thief'], $a a_{3}$ is in the referential layer of the nominal projection as it cannot have a non-referential reading. In context where familiarity towards a thief is called for, $a a_{3}$ can be added. But this kind of familiarity is contextual rather than lexicalized, just like in multisyllabic aa3-nominals. This means it is possible to add $a a_{3}$ to monosyllabic [+human] nouns that are not [+familiar] lexically, given an appropriate context. Surprisingly, in these cases, modification seems to be more acceptable:

(6o) hou2 goul ge3 aa3-caak2

very tall GE ah-thief

This seems to suggest that nominals like [aa3-caak2 'thief'] behave in a way that is in-between disyllabic aa3-nominals (as we have discussed so far) and multisyllabic aa3-nominals. Caak2 'thief' is not lexically [+familiar] thus aa3 is not interpreted as part of the lexical item and thus it cannot follow a classifier and be non-referential. On the other hand, it is disyllabic and thus has the same shape as a minimal prosodic unit, and could be interpreted as a lexical item (Feng 2009). The outcome is such that it is incompatible with classifiers but is compatible with modifiers. This seems to reveal some interesting interaction between prosody and syntax, which we shall leave for further research.

\section{Acknowledgements}

We would like to thank the anonymous reviewers for their valuable comments and the editorial team for their help in the whole submission process. All errors remain our own. The work was supported by European Regional Development Fund-Project "Sinophone Borderlands - Interaction at the Edges" CZ.02.1.01/o.o/o.o/16_019/00oo791.

\section{References}

Abbott, Barbara. 2002. Discussion Note: Definiteness and proper names: Some bad news for descriptive theory. Journal of Semantics 19(2). 191-201. https://doi.org/10.1093/jos/19.2.191

Bauer, Robert S., \& Benedict, Paul. 1997. Modern cantonese phonology. Berlin: Mouton de Gruyter. https://doi.org/10.1515/9783110823707

Brown, Roger \& Gilman, Albert. 1960. The pronouns of power and solidarity. In Sebeok, Thomas A. (ed.), Style in language, 253-276. Cambridge: The MIT Press.

Chao, Yuen Ren. 1947. Cantonese primer. Cambridge: Harvard University Press. https://doi.org /10.4159/harvard.9780674732438

Chao, Yuen Ren. 1968. A grammar of spoken Chinese. Berkeley: University of California Press. 
Cheng, Lisa Lai-Shen. 1990. Feature geometry of vowels and co-occurrence restrictions in Cantonese. (Paper presented at the West Coast Conference on Formal Linguistics 9, Stanford, 2-4 March 1990.)

Cheng, Lisa Lai-Shen \& Sybesma, Rint. 1999. Bare and not-so-bare nouns and the structure of NP. Linguistic Inquiry 30(4). 509-542. https://doi.org/10.1162/002438999554192

Di Sciullo, Anna-Maria \& Williams, Edwin. 1987. On the definition of word. Cambridge: The MIT Press.

Feng, Shengli. 2009. Minimal word and its function in Mandarin Chinese. In Xing, Janet Zhiqun (ed.), Studies of Chinese linguistics: Functional approaches, 47-64. Hong Kong: Hong Kong University Press.

Geurts, Bart. 1997. Good news about the description theory of names. Journal of Semantics 14(4). 319-348. https://doi.org/10.1093/jos/14.4.319

Heim, Irene Roswitha. 1982. The semantics of definite and indefinite noun phrases. Amherst: University of Massachusetts. (Doctoral dissertation.)

Hudson, Richard A. 1996. Sociolinguistics. 2nd edition. Cambridge: Cambridge University Press. https://doi.org/10.1017/CBO9781139166843

Huang, Borong (ed.). 1996. Hanyu fangyan yufa lei bian [Classified materials on Chinese dialect grammar], Qingdao: Qingdao Publishing House.

Longobardi, Giuseppe. 1994. Reference and proper names: A theory of N-movement in syntax and logical form. Linguistic Inquiry 25(4). 609-665.

Lyons, Christopher. 1999. Definiteness. Cambridge: Cambridge University Press. https://doi.org /10.1017/CBO9780511605789

Matthews, Stephen \& Yip, Virginia. 2011. Cantonese: A comprehensive grammar. 2nd edn. London: Routledge.

Prince, Ellen F. 1992. The ZPG letter: Subjects, definiteness, and information status. In Mann, William C. \& Thompson, Sandra A. (eds.), Discourse description: Diverse linguistic analyses of a fund-raising text, 295-326. Amsterdam: John Benjamins. https://doi.org/10.1075/pbns .16.12pri

Sio, Joanna Ut-Seong. 2003. Two types of possessive constructions in Cantonese. In Lin, YenHwei (ed.), Proceedings of the Fifteen North American Conference on Chinese Linguistics, 313-330. Los Angeles: GSIL Publications, University of Southern California.

Sio, Joanna Ut-Seong. 2006. Modification and reference in the Chinese nominal. Utrecht: LOT Publications.

Sio, Joanna Ut-Seong. 2010. Syntax of [+human] terms in Cantonese. In Wilder, Chris \& Åfarli, Tor Anders (eds.), Chinese matters: From grammar to first and second language acquisition, 15-27. Norway: Tapir Academic Press.

Sybesma, Rint \& Li, Boya. 2007. The dissection and structural mapping of Cantonese sentence final particles. Lingua 117(10). 1739-1783. https://doi.org/10.1016/j.lingua.2006.10.003

Tang, Sze-Wing. 2011. A parametric approach to NP ellipsis in Mandarin and Cantonese. Journal of East Asian Linguistics 20(2). 107-115. https://doi.org/10.1007/s10831-010-9067-7

Tang, Sze-Wing. 2015. Yueyu yufa jiangyi [Lectures on Cantonese grammar]. Hong Kong: The Commercial Press.

Yip, Moira. 1992. Prosodic morphology in four Chinese dialects. Journal of East Asian Linguistics. 1(1). 1-35. https://doi.org/10.1007/BFoo129572

Zhan, Bohui (ed.). 2002. Guangdong Yue fangyan gaiyao [An outline of Yue Dialects in Guangdong]. Guangzhou: Jinan University Press. 


\section{Authors' addresses}

Joanna Ut-Seong Sio (corresponding author)

Department of Asian Studies

Palacký University

Křížkovského 8

Olomouc 77180

Czech Republic

joannautseong.sio@upol.cz

\section{Publication history}

Date received: 5 January 2016

Date accepted: 31 January 2018

Published online: 2 January 2020 